\title{
SYNCHRONIZATION OF GENESIO AND LÜ CHAOTIC DYNAMICAL SYSTEMS VIA ACTIVE CONTROL
}

\author{
Ayub Khan ${ }^{1}$, Dinesh Khattar ${ }^{2}$, Neha Agrawal $^{3} \S$ \\ ${ }^{1}$ Department of Mathematics \\ Jamia Millia Islamia University \\ New Delhi, 110025, INDIA \\ ${ }^{2,3}$ Department of Mathematics \\ Kirori Mal College \\ University of Delhi \\ Delhi, 110007, INDIA
}

\begin{abstract}
In this paper we have investigated Complete Synchronization, Anti Synchronization and Hybrid Synchronization between the Genesio chaotic dynamical system and Lü chaotic dynamical system using nonlinear active control technique based on Lyapunov Stability theory. Further numerical simulations are carried out to show the effectiveness of the method.
\end{abstract}

AMS Subject Classification: 34D06, 34H10, 34D08

Key Words: synchronization, Genesio system, Lü system, active control

\section{Introduction}

Chaos is an interesting nonlinear phenomenon and has been intensively studied in the last three decades. Chaos theory has been applied in many scientific disciplines such as Mathematics, Economics, Microbiology, Biology, Ecology, Computer Science and Robotics.

Received: November 25, 2015

(c) 2016 Academic Publications

$\S_{\text {Correspondence author }}$ 
A chaotic system is a special non linear dynamical system, which has many properties such as the sensitivity to initial conditions as well as an irregular, unpredictable behaviour. During the last few years synchronization in chaotic dynamical systems has received a great deal of attention for the scientists from various fields $[2,5,6,18,41]$.

In 1990, Pecora and Carroll developed control techniques to synchronize two identical chaotic systems and showed that it was possible for some chaotic systems to be completely synchronized. From then on, chaos synchronization has emerged as an important area of research in many fields such as physical systems [7, 20], chemical systems [11, 25], ecological systems [3] and secure communication $[4,19,38]$ etc.

A wide variety of approaches has been used for the synchronization of chaotic systems such as active control method [1, 12, 13, 17, 32], adaptive control [23, 36], backstepping design [37, 39], OGY method [26], sampled- data feedback synchronization method [24, 44], time delay feedback method [28], sliding mode control method [31, 35] and PC method [29], etc. However most of these methods synchronize two identical chaotic systems. But in many practical problems such as laser array and biological systems, it is hardly the case that every component can be assumed to be identical. More and more applications of chaos synchronization in secure communication make it even more important to synchronize two different chaotic systems. In recent years, some works on synchronization of two different chaotic systems have been performed $[12,27,42]$. So far many Synchronization phenomenon have been presented such as complete synchronization [16], phase synchronization [8], anti synchronization [16, 33, 43], generalized synchronization [40], hybrid synchronization $[14,15,16,34]$, projective synchronization [30] and generalized projective synchronization [21], etc.

In this paper we study the chaos synchronization between two different chaotic systems using active nonlinear control method. The stability results derived in this paper are established using Lyapunov stability theory. The Genesio system is taken as the master system and the Lü system as the slave system. This paper is organized as follows. In Section 2, we provide a description of the chaotic systems. In Section 3, we discuss the Complete synchronization, Anti synchronization and Hybrid synchronization between the Genesio chaotic system and the Lü chaotic dynamical system using active control. In Section 4, we discuss the numerical simulations. 


\section{System Description}

The master system is described by the Genesio system, proposed by Genesio et al. $[9,32]$ capturing many features of chaotic systems. It includes three simple ordinary differential equations that depend on three positive real parameters. The dynamical equations are given by

$$
\left.\begin{array}{l}
\dot{x_{1}}=x_{2}, \\
\dot{x_{2}}=x_{3}, \\
\dot{x_{3}}=-\mathrm{c} x_{1}-\mathrm{b} x_{2}-\mathrm{a} x_{3}+x_{1}^{2},
\end{array}\right\}
$$

where $x_{1}, x_{2}$ and $x_{3}$ are state variables and $a, b$ and $c$ are positive constants satisfying $a b<c$. The system exhibits a chaotic behaviour for the parameters values $a=1.2, b=2.92$ and $c=6$, shown in Fig. 1 .

The slave system is described by the Lü system, [22] is a typical transition system, which connects the Lorenz and Chen attractors and represents the transition from one to the other,that captures many features of chaotic systems. The dynamical equations are given by

$$
\left.\begin{array}{l}
\dot{y_{1}}=\alpha\left(y_{2}-y_{1}\right), \\
\dot{y_{2}}=\gamma y_{2}-y_{1} y_{3}, \\
\dot{y_{3}}=-\beta y_{3}+y_{1} y_{2},
\end{array}\right\}
$$

where $y_{1}, y_{2}$ and $y_{3}$ are state variables and $\alpha, \beta$ and $\gamma$ are positive constants. The system exhibits a chaotic behaviour for the parameters values $\alpha=36, \beta$ $=3$ and $\gamma=20$ as shown Fig. 2 . 


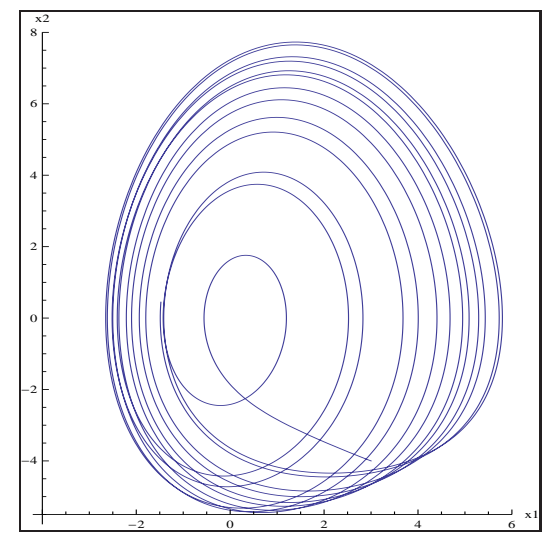

Fig.1 Genesio chaotic system with

$a=1.2, b=2.92$ and $c=6$

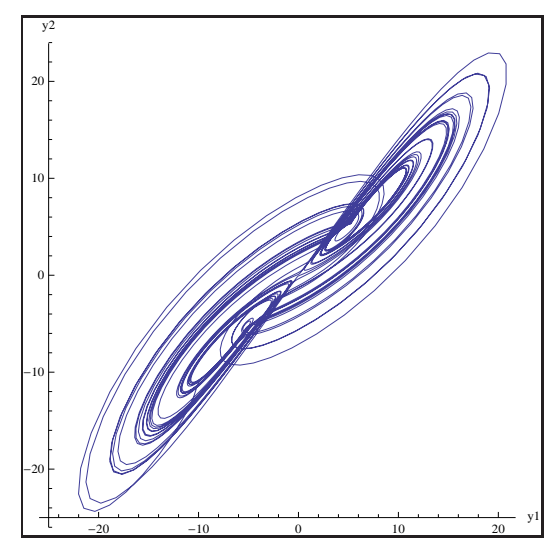

Fig.2 Lü chaotic system with $\alpha=36, \beta=3$ and $\gamma=20$

3. Complete Synchronization, Anti Synchronization and Hybrid Synchronization between Genesio Chaotic System and Lü Chaotic System

\subsection{Complete Synchronization}

Complete synchronization is characterized by the equality of state variables evolving in time. As the master system we consider the Genesio dynamical system defined by

$$
\left.\begin{array}{l}
\dot{x_{1}}=x_{2}, \\
\dot{x_{2}}=x_{3}, \\
\dot{x_{3}}=-\mathrm{c} x_{1}-\mathrm{b} x_{2}-\mathrm{a} x_{3}+x_{1}^{2},
\end{array}\right\}
$$

where $x_{1}, x_{2}$ and $x_{3}$ are state variables and $a, b$ and $c$ are unknown, real, constant parameters satisfying $a b<c$.

As the slave system, we consider the Lü dynamical system described by

$$
\left.\begin{array}{l}
\dot{y_{1}}=\alpha\left(y_{2}-y_{1}\right)+u_{1}, \\
\dot{y_{2}}=\gamma y_{2}-y_{1} y_{3}+u_{2}, \\
\dot{y_{3}}=-\beta y_{3}+y_{1} y_{2}+u_{3},
\end{array}\right\}
$$


where $y_{1}, y_{2}$ and $y_{3}$ are state variables, $\alpha, \beta$ and $\gamma$ are unknown, real, constant parameters and $u_{1}, u_{2}$ and $u_{3}$ are control functions to be designed. The synchronization errors $e_{i}, i=1,2,3$ are defined by $e_{i}=y_{i}-x_{i}$. From (3) and (4), we obtain the error dynamics as follows:

$$
\left.\begin{array}{l}
\dot{e_{1}}=\alpha\left(y_{2}-y_{1}\right)-x_{2}+u_{1}, \\
\dot{e_{2}}=\gamma y_{2}-y_{1} y_{3}-x_{3}+u_{2}, \\
\dot{e_{3}}=-\beta y_{3}+y_{1} y_{2}+\mathrm{c} x_{1}+\mathrm{b} x_{2}+\mathrm{a} x_{3}-x_{1}^{2}+u_{3} .
\end{array}\right\}
$$

We choose the nonlinear controllers as

$$
\left.\begin{array}{l}
u_{1}=-\alpha\left(y_{2}-y_{1}\right)+x_{2}-k_{1} e_{1}, \\
u_{2}=-\gamma y_{2}+y_{1} y_{3}+x_{3}-k_{2} e_{2}, \\
u_{3}=\beta y_{3}-y_{1} y_{2}-\mathrm{c} x_{1}-\mathrm{b} x_{2}-\mathrm{a} x_{3}+x_{1}^{2}-k_{3} e_{3},
\end{array}\right\}
$$

where $k_{1}, k_{2}$ and $k_{3}$ are positive constants. Substituting (6) into (5), we get

$$
\left.\begin{array}{r}
\dot{e_{1}}=-k_{1} e_{1} \\
\dot{e_{2}}=-k_{2} e_{2} \\
\dot{e_{3}}=-k_{3} e_{3}
\end{array}\right\}
$$

Consider the Lyapunov function defined by

$$
V(e)=\frac{1}{2}\left(e_{1}^{2}+e_{2}^{2}+e_{3}^{2}\right) .
$$

Differentiating (8) along the trajectories of (7) we get

$$
\dot{V}(e)=-k_{1} e_{1}^{2}-k_{2} e_{2}^{2}-k_{3} e_{3}^{2}
$$

which is a negative definite function on $\mathbb{R}^{2}$. Thus by Lyapunov stability theory [10], the error dynamics (7) is found to be asymptotically stable and converges to zero exponentially with time and thus complete synchronization is achieved between master system (3) and slave system (4). Hence the non-identical Genesio system (3) and Lü system (4) are exponentially and globally synchronized using nonlinear active control law. 


\subsection{Anti Synchronization}

Anti synchronization is characterized by the disappearance of the sum of relevant variables evolving in time. To observe anti synchronization between the master system and the slave system, we first define the synchronization errors $e_{i}, i=1,2,3$ by $e_{i}=y_{i}+x_{i}$. From (3) and (4), we obtain the error dynamics as follows:

$$
\left.\begin{array}{l}
\dot{e_{1}}=\alpha\left(y_{2}-y_{1}\right)+x_{2}+u_{1}, \\
\dot{e_{2}}=\gamma y_{2}-y_{1} y_{3}+x_{3}+u_{2}, \\
\dot{e_{3}}=-\beta y_{3}+y_{1} y_{2}-\mathrm{c} x_{1}-\mathrm{b} x_{2}-\mathrm{a} x_{3}+x_{1}^{2}+u_{3} .
\end{array}\right\}
$$

We choose the nonlinear controllers as

$$
\left.\begin{array}{l}
u_{1}=-\alpha\left(y_{2}-y_{1}\right)-x_{2}-k_{1} e_{1}, \\
u_{2}=-\gamma y_{2}+y_{1} y_{3}-x_{3}-k_{2} e_{2}, \\
u_{3}=\beta y_{3}-y_{1} y_{2}+\mathrm{c} x_{1}+\mathrm{b} x_{2}+\mathrm{a} x_{3}-x_{1}^{2}-k_{3} e_{3},
\end{array}\right\}
$$

where $k_{1}, k_{2}$ and $k_{3}$ are positive constants. Substituting (11) into (10), we get

$$
\left.\begin{array}{c}
\dot{e_{1}}=-k_{1} e_{1} \\
\dot{e_{2}}=-k_{2} e_{2} \\
\dot{e_{3}}=-k_{3} e_{3}
\end{array}\right\}
$$

Consider the Lyapunov function defined by

$$
V(e)=\frac{1}{2}\left(e_{1}^{2}+e_{2}^{2}+e_{3}^{2}\right) .
$$

Differentiating (13) along the trajectories of (12) we get

$$
\dot{V}(e)=-k_{1} e_{1}^{2}-k_{2} e_{2}^{2}-k_{3} e_{3}^{2}
$$

which is a negative definite function on $\mathbb{R}^{2}$. Thus by Lyapunov stability theory [10], the error dynamics (12) is found to be asymptotically stable and converges to zero exponentially with time and hence anti synchronization is achieved between master system (3) and slave system (4).

Thus the non-identical Genesio system (3) and Lü system (4) are exponentially and globally anti synchronized for all initial conditions using nonlinear controller defined by (11). 


\subsection{Hybrid Synchronization}

In Hybrid synchronization of chaotic systems, one part of the system is synchronized and the other part is anti synchronized so that complete synchronization and anti synchronization exist together in the system. This coexistence of complete synchronization and anti synchronization is useful in secure communication and chaotic encryption schemes. The Hybrid synchronization errors are defined by

$$
\left.\begin{array}{l}
e_{1}=y_{1}-x_{1}, \\
e_{2}=y_{2}+x_{2}, \\
e_{3}=y_{3}-x_{3} .
\end{array}\right\}
$$

Here, we see that one part of the two chaotic systems is completely synchronized and the other part is anti synchronized so that complete synchronization and anti synchronization exist together in the synchronization of systems (3) and (4).

From (3) and (4) we obtain the error dynamics as follows:

$$
\left.\begin{array}{l}
\dot{e_{1}}=\alpha\left(y_{2}-y_{1}\right)-x_{2}+u_{1}, \\
\dot{e_{2}}=\gamma y_{2}-y_{1} y_{3}+x_{3}+u_{2}, \\
\dot{e_{3}}=-\beta y_{3}+y_{1} y_{2}+\mathrm{c} x_{1}+\mathrm{b} x_{2}+\mathrm{a} x_{3}-x_{1}^{2}+u_{3} .
\end{array}\right\}
$$

We choose the nonlinear controllers as

$$
\left.\begin{array}{l}
u_{1}=-\alpha\left(y_{2}-y_{1}\right)+x_{2}-k_{1} e_{1}, \\
u_{2}=-\gamma y_{2}+y_{1} y_{3}-x_{3}-k_{2} e_{2}, \\
u_{3}=\beta y_{3}-y_{1} y_{2}-\mathrm{c} x_{1}-\mathrm{b} x_{2}-\mathrm{a} x_{3}+x_{1}^{2}-k_{3} e_{3},
\end{array}\right\}
$$

where $k_{1}, k_{2}$ and $k_{3}$ are positive constants. Substituting (17) into (16), we get

$$
\left.\begin{array}{l}
\dot{e_{1}}=-k_{1} e_{1}, \\
\dot{e_{2}}=-k_{2} e_{2}, \\
\dot{e_{3}}=-k_{3} e_{3} .
\end{array}\right\}
$$

Consider the Lyapunov function defined by

$$
V(e)=\frac{1}{2}\left(e_{1}^{2}+e_{2}^{2}+e_{3}^{2}\right) .
$$


Differentiating (19) along the trajectories of (18), we get

$$
\dot{V}(e)=-k_{1} e_{1}^{2}-k_{2} e_{2}^{2}-k_{3} e_{3}^{2}
$$

which is a negative definite function on $\mathbb{R}^{2}$. Thus by Lyapunov stability theory [10], the hybrid synchronization errors $e_{i}, i=1,2,3$ converges to zero exponentially with time. In the similar manner, the 5 other hybrid synchronization phenomena can be described. We find that in all the cases after defining the control functions $u_{i}, i=1,2,3$ the linear error system obtained is identical to the one defined in (18). Hence the hybrid synchronization is attainable in all the cases. Thus the non-identical Genesio system (3) and Lü system (4) are exponentially and globally hybrid synchronized using nonlinear active control law.

\section{Numerical Simulations}

For simulations, the parameters of the Genesio Chaotic system are chosen as $a=1.2, b=2.92$ and $c=6$. The parameters of Lü chaotic system are chosen as $\alpha=36, \beta=3$ and $\gamma=20$. The initial conditions of the master and slave system are chosen as $x(0)=(3,-4,2)$ and $y(0)=(-10,-11,5)$, respectively. For complete synchronization, the control functions defined by (6) are used and the systems (3) and (4) are simulated, for anti synchronization, the control functions defined by (11) are used and the systems (3) and (4) are simulated and for hybrid synchronization, the control functions defined by (17) are used and the systems (3) and (4) are simulated. The results of the simulation of the two non identical chaotic systems without active control are shown in Fig. 3: (a) displays the $x_{1}$ and $y_{1},(b)$ displays the $x_{2}$ and $y_{2}$, and $(c)$ displays the $x_{3}$ and $y_{3}$, where $x_{1}, x_{2}$ and $x_{3}$ are state variables of the Genesio Chaotic system and $y_{1}, y_{2}$ and $y_{3}$ are state variables of the Lü chaotic system. Fig. 4 (a) - (c) display the same sequence of signals, when completely synchronized using the active control. The corresponding time series analysis graph of the synchronization errors are given in Fig. $5(a)-(c)$. 


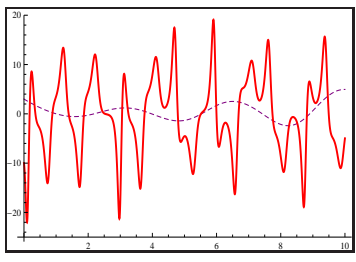

Fig.3 (a) State trajectories $x_{1}(--)$ and $y_{1}(-)$ without active controllers

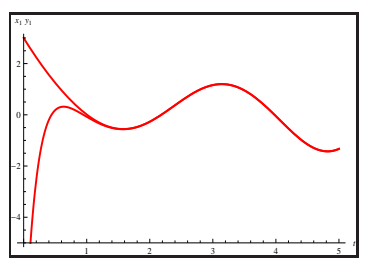

Fig. 4 (a) State trajectories $x_{1}$ and $y_{1}$ with active controllers

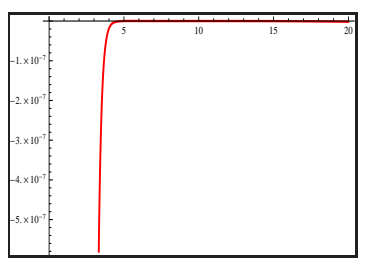

Fig.5 (a) Time series analysis of $e_{1}(t)$
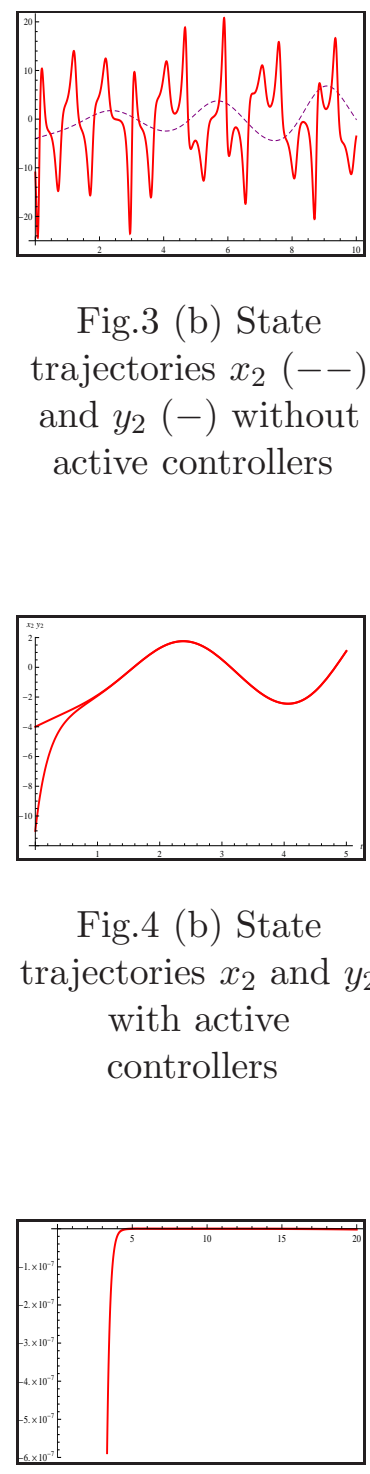

Fig.3 (b) State trajectories $x_{2}(--)$ and $y_{2}(-)$ without active controllers

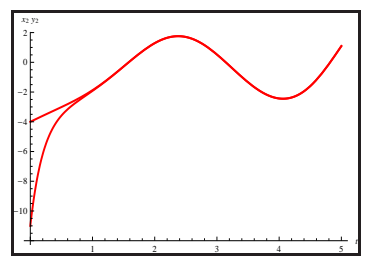

Fig.4 (b) State trajectories $x_{2}$ and $y_{2}$ with active controllers

Fig.5 (b) Time series analysis of $e_{2}(t)$

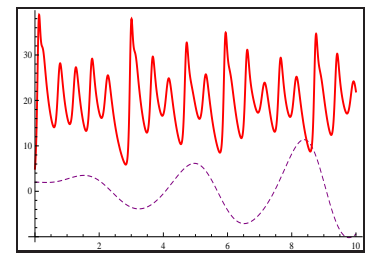

Fig.3 (c) State trajectories $x_{3}(--)$ and $y_{3}(-)$ without active controllers

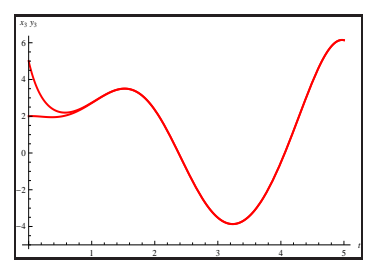

Fig.4 (c) State trajectories $x_{3}$ and $y_{3}$ with active controllers

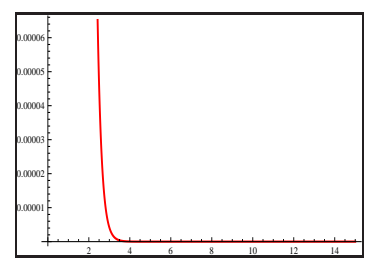

Fig.5 (c) Time series analysis of $e_{3}(t)$

Fig. $6(a)-(c)$ display the same sequence of signals, when the signals are anti synchronized using the active control. The corresponding time series analysis graph of the synchronization errors are given in Fig. $7(a)-(c)$. 


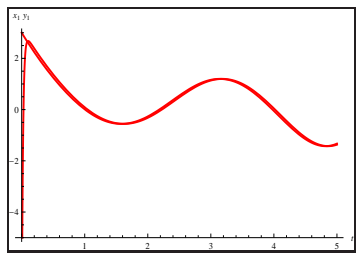

Fig.6 (a) State trajectories $x_{1}$ and $y_{1}$ with active controllers

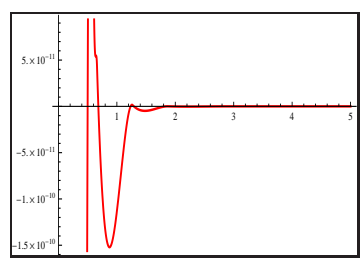

Fig.7 (a) Time series analysis of $e_{1}(t)$

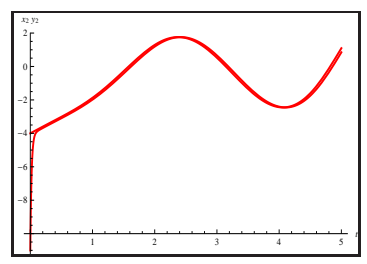

Fig.6 (b) State with active controllers

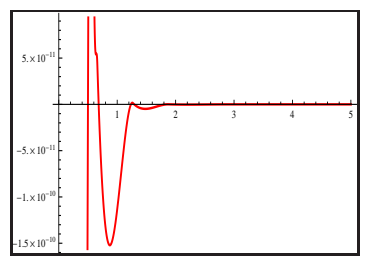

Fig.7 (b) Time series analysis of $e_{2}(t)$

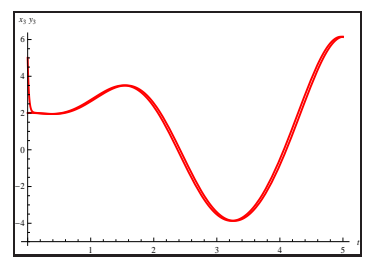

Fig.6 (c) State trajectories $x_{3}$ and $y_{3}$ with active controllers

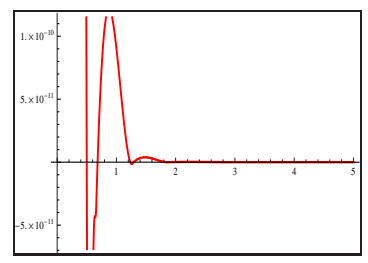

Fig.7 (c) Time series analysis of $e_{3}(t)$

Fig. $8(a)-(c)$ display the same sequence of signals, when the signals are hybrid synchronized using the active control. The corresponding time series analysis graph of the synchronization errors are given in Fig. $9(a)-(c)$.

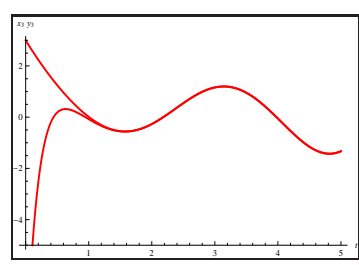

Fig.8 (a) State trajectories $x_{1}$ and $y$ with active controllers

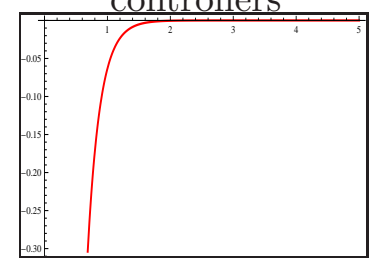

Fig.9 (a) Time series analysis of $e_{1}(t)$

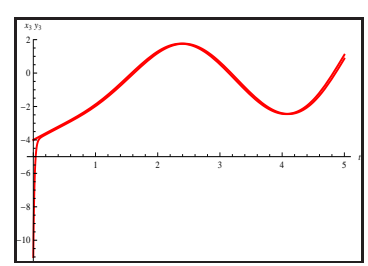

Fig.8 (b) State trajectories $x_{2}$ and $y_{2}$ with active controllers

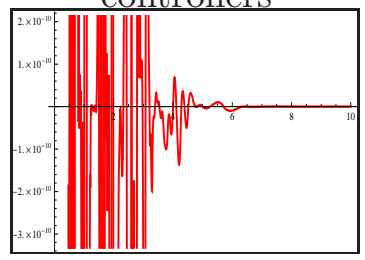

Fig.9 (b) Time series analysis of $e_{2}(t)$

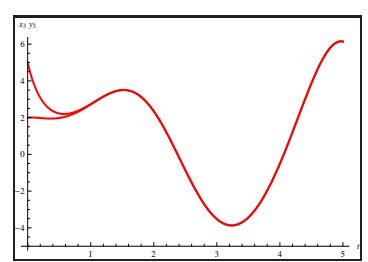

Fig.8 (c) State trajectories $x_{3}$ and $y_{3}$ with active controllers

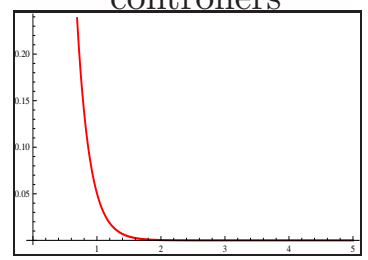

Fig.9 (c) Time series analysis of $e_{3}(t)$ 


\section{Conclusion}

In this paper, we have investigated synchronization behavior between the Genesio chaotic system and the Lü chaotic system via active control method and Lyapunov stability theory. Since the Lyapunov exponents are not needed for these calculations, the nonlinear control method is very effective and convenient to achieve global chaos complete synchronization, anti synchronization and hybrid synchronization between the systems studied in this paper. The results are validated by numerical simulations using Mathematica and Matlab both.

\section{References}

[1] H.N. Agiza and M.T. Yassen, Synchronization of Rossler and Chen chaotic dynamical systems using active control, Phys Lett. A, 278 (2001), 191-197.

[2] E. Bai and K.E. Lonngrn, Sequential synchronization of two Lorenz systems using active control, Chaos Solitons Fractals, 11, No 7 (2000), 10411044 .

[3] B. Blasius, A. Huppert and L. Stone, Complex dynamics and phase synchronization in spatially extended ecological system, Nature, 399 (1999), 354-359.

[4] S. Boccaletti, A. Farini and F.T. Arecchi, Adaptive synchronization of chaos for secure communication, Physical Review E, 55, No 5 (1997), 49794981.

[5] T.L. Carroll and L.M. Pecora, Synchronizing chaotic circuits, IEEE Trans. Circuits Syst. I, 38 (1991), 453-456.

[6] G. Chen and X. Dong, On feedback control of chaotic continuous-time systems, IEEE Trans. Circuits Syst., 40 (1993), 591-601.

[7] L. Fabiny and K. Wiesenfield, Clustering behaviour of oscillator arrays, Physical Review A, 43 (1991), 2640-2648.

[8] Z.M. Ge and C.C. Chen, Phase synchronization of coupled chaotic multiple time scales systems, Chaos, Solitons and Fractals, 20 (2004), 639-647.

[9] R. Genesio and A. Tesi, A harmonic balance methods for the analysis of chaotic dynamics in nonlinear systems, Automatica, 28 (1992), 531-548. 
[10] W. Hahn, The Stability of Motion, Springer, Berlin (1967).

[11] S.K. Han, C. Kerrer and Y. Kuramoto, Dephasing and bursting in coupled neural oscillators, Physical Review Letters, 75 (1995), 3190-3193.

[12] M.C. Ho and Y.C. Hung, Synchronization of two different systems by using generalized active control, Phys. Lett. A, 301 (2002), 424-428.

[13] L. Huang, R. Feng and M. Wang, Synchronization of chaotic systems via nonlinear control, Physical Letters A, 320 (2005), 271-275.

[14] X. Huang, Z. Wang and Y. Li, Hybrid synchronization of hyperchaotic Lü system based on passive control, International Workshop on Chaos-Fractal, Theories and Applications, Kunming, Yunnan (2010), 34-38.

[15] H.C. Juhn, K.C. Hsien and K.L. Yu, Synchronization and anti synchronization coexist in Chen-Lee chaotic systems, Chaos, Solitons and Fractals, 39, No 2 (2009), 707-716.

[16] A. Khan and R. Pal, Complete synchronization, anti synchronization and Hybrid synchronization of two identical parabolic restricted three body problem, Asian Journal of Current Engineering and Maths, 2 (2013), 118126.

[17] M.A. Khan, Synchronization of different 3D chaotic systems by generalized active control, Journal of Information and Computing Science, 7, No 4 (2012), 272-283.

[18] L. Kocarev, K.S. Halle, K. Eckert, L.O. Chua and U. Parlitz, Experimental demonstration of secure communications via chaotic synchronization, Int. J. of Bifurcation and Chaos, 2, No 3 (1992), 709-713.

[19] L. Kocarev and U. Parlitz, General approach for chaotic synchronization with applications to communication, Physical Review Letters, 74 (1995), 5028-5030.

[20] M. Lakshmanan and K. Murali, Nonlinear Oscillators: Controlling and Synchronization, World Scientific, Singapore (1996).

[21] R.H. Li, W. Xu and S. Li, Adaptive generalized projective synchronization in different chaotic systems based on parameter identification, Phys. Lett. A, 367 (2007), 199-206. 
[22] J. Lu and G.R. Chen, A new chaotic attractor coined, Int. Journal Bifurcat Chaos, 12 (2002), 659-661.

[23] J. Lu, X. Wu, X. Han and J. Lü, Adaptive feedback synchronization of a unified chaotic system, Physics Letters A, 329 (2004), 327-333.

[24] K. Murali and M. Lakshmanan, Secure communication using a compound signal using sampled- data feedback, Applied Mathematics and Mechanics, 11 (2003), 1309-1315.

[25] H. Niu, Q. Zhang and Y. Zhang, The chaos synchronization of singular chemical model and a Williamowski-Rossler model, International Journal of Information and Systems Sciences, 6, No 4 (2002), 355-364.

[26] E. Ott, C. Grebogi and J.A. Yorke, Controlling chaos, Phys. Rev. Lett., 64 (1990), 1196-1199.

[27] J.H. Park, Chaos synchronization between two different chaotic dynamical systems, Chaos, Solitons and Fractals, 27 (2006), 549-554.

[28] J.H. Park and O.M. Kwon, A novel criterion for delayed feedback control of time-delay chaotic systems, Chaos, Solitons and Fractals, 17 (2003), 709-716.

[29] L.M. Pecora and T.L. Carroll, Synchronization in chaotic systems, Phys. Rev. Lett., 64 (1990), 821-824.

[30] J. Qiang, Projective synchronization of a new hyperchaotic Lorenz system, Phys. Lett. A, 370 (2007), 40-45.

[31] Z. Rabiei, G.R. Bidari and N. Pariz, Synchronization between two different chaotic systems using adaptive sliding mode control, International Journal of Information and Electronics Engineering, 3, No 1 (2013), 83-86.

[32] M. Shahzad, Chaos synchronization of an ellipsoidal satellite via active control, Progress in Applied Mathematics, 3 (2002), 16-23.

[33] J.P. Singh, P.P. Singh and B.K. Roy, Anti-synchronization of BhalekarGejji chaotic system via nonlinearactive control, STM Journals (2014), 112-117.

[34] V. Sundarapandian, Hybrid synchronization of Liu and Lü chaotic systems via adaptive control, International Journal of Advanced Information Technology, 1, No 6 (2011), 13-32. 
[35] V. Sundarapandian, Sliding mode controller design for synchronization of Shimizu-Morioka chaotic systems, International Journal of Information Sciences and Techniques, 1, No 1 (2011), 20-29.

[36] V. Sundarapandian, Adaptive synchronization of hyperchaotic Lorenz and hyperchaotic Lü systems, International Journal of Instrumentation and Control Systems, 1, No 1 (2011), 1-18.

[37] X. Tan, J. Zhang and Y. Yang, Synchronizing chaotic systems using back stepping design, Chaos, Solitons and Fractals, 16 (2003), 37-45.

[38] Y. Tao, Chaotic secure communication systems- history and new results, Telecommun. Review, 9 (1999), 597-634.

[39] U.E. Vincent, Chaotic synchronization using active control and back stepping control: A comparative analysis, Nonlinear Analysis: Modelling and Control, 13, No 2 (2008), 253-261.

[40] Y.W. Wang and Z.H. Guan, Generalized synchronization of continuous chaotic systems, Chaos, Solitons and Fractals, 27 (2006), 97-101.

[41] Q. Xie and G. Chen, Synchronization stability analysis of the chaotic Rössler system, Int. J. Bifurcation and Chaos, 6 (1996), 2153-2161.

[42] M.T. Yassen, Chaos synchronization between two different chaotic systems using active control, Chaos, Solitons and Fractals, 23 (2005), 131-140.

[43] X. Zhang and H. Zhu, Anti-synchronization of two different hyperchaotic systems via active and adaptive control, International Journal of Nonlinear Science, 6, No 3 (2008), 216-223.

[44] J. Zhao and J. Lu, Using sampled-data feedback control and linear feedback synchronization in a new hyper chaotic system, Chaos, Solitons and Fractals, 35 (2006), 376-382. 